October 2019

\title{
Sasakians and the Geometry of a Mass Term
}

\author{
V.P. NAIR \\ Physics Department, City College of the CUNY \\ New York, NY 10031 \\ E-mail: vpnair@ccny.cuny.edu
}

\begin{abstract}
A gauge-invariant mass term for nonabelian gauge fields in two dimensions can be expressed as the Wess-Zumino-Witten (WZW) action. Hard thermal loops in the gauge theory in four dimensions at finite temperatures generate a screening mass for some components of the gauge field. This can be expressed in terms of the WZW action using the bundle of complex structures (for Euclidean signature) or the bundle of lightcones over Minkowski space. We show that a dynamically generated mass term in three dimensions can be put within the same general framework using using the bundle of Sasakian structures.
\end{abstract}

Dedicated to Roman Jackiw on his 80th Birthday

To appear in "Roman Jackiw: 80th Birthday Festschrift", edited by A. Niemi, T. Tomboulis, and K. K. Phua (World Scientific, 2020) 


\section{Introduction}

The early 1980s were a time of growing appreciation of the role of topology in quantum field theory, especially for gauge theories. Anomalies and Chern-Simons terms were very much in the air, so it was impossible for any graduate student to be unaware of the seminal contributions of Roman Jackiw. My own collaboration with Roman began somewhat later, during his sabbatical visit to Columbia University in 1990. At that time Roman was very much interested in solitons in Chern-Simons theories coupled to matter fields, both relativistic and nonrelativistic [1], but we did talk about anomalies and anyons and representations of the Poincare group in $2+1$ dimensions. After he went back to MIT, we corresponded about anyons, and this evolved into our paper on the relativistic wave equation for anyons [2].

Throughout the 1990s and early 2000s, we continued to collaborate on a number of projects of common interest, from finite temperature field theories, nonabelian Clebsch parametrization [3], a group theory based formulation of nonabelian magnetohydrodynamics [4], etc. Particularly gratifying was my work with Efraty on an effective action for hard thermal loops [5], and the subsequent work with Roman on developing it into a nonabelian version of the Kubo formula [6], combining two of his favorite topics: field theory at finite temperature and Chern-Simons theory. Chromomagnetic screening masses and gap equations in $2+1$ dimensional gauge theories was another topic on which I had many discussions with Roman and So-Young, although we never published any joint work on this [7]. Looking back, it is striking to me that we had overlap of interest on so many different topics. Yet, on second thought, it is perhaps not so remarkable since Roman has been a continuing influence on the development of field theory from the mid-1960s to the present, and hence any one interested in field theory would be bound to have many points of overlap with his work.

To a man who has devoted decades to physics, appreciation must be shown in kind, not just in anecdotes and reminiscences alone. So, for my contribution to this Festschrift, I have decided to write on a novel aspect of something we have both worked on. I shall discuss dynamically generated mass terms in gauge theories, which brings together Chern-Simons actions and their eikonals, the Wess-Zumino-Witten actions, Dirac determinants, chromomagnetic screening effects and many facets of geometry and topology, which are all topics of interest to Roman. The key point is that while Kähler structures play an important role for physics in even dimensions, Sasakian structures should do

so in odd dimensions. A mass term which can be dynamically generated in nonabelian gauge theories in odd dimensions, as I argue below, exemplifies this. 


\section{Masses for gauge theories in two and four dimensions}

The prototypical example of a gauge-invariant mass term is given by the Wess-ZuminoWitten (WZW) action in two dimensions [8], or, equivalently, by the logarithm of the Dirac determinant [9]. This is the nonabelian generalization of Schwinger's result for the Abelian case [10]. Specifically, this mass term takes the form

$$
\begin{aligned}
\Gamma= & -[\operatorname{Tr} \log (-\bar{D} D)-\operatorname{Tr} \log (-\bar{\partial} \partial)]=-A_{R} S_{\mathrm{WZW}}(H) \\
S_{\mathrm{WZW}}(H)= & \frac{1}{2 \pi} \int_{\mathcal{M}} d^{2} z \operatorname{Tr}\left(\partial H \bar{\partial} H^{-1}\right) \\
& +\frac{i}{12 \pi} \int_{\mathcal{M}^{3}} \operatorname{Tr}\left(H^{-1} d H \wedge H^{-1} d H \wedge H^{-1} d H\right)
\end{aligned}
$$

where we use complex coordinates in two dimensions, $z, \bar{z}=x_{1} \mp i x_{2}$ and

$$
\begin{aligned}
& D=\partial+A=\frac{1}{2}\left(\partial_{1}+i \partial_{2}\right)+\frac{1}{2}\left(A_{1}+i A_{2}\right)=\frac{1}{2}\left(\partial_{1}+i \partial_{2}\right)+\left(-i t_{a}\right) \frac{1}{2}\left(A_{1}^{a}+i A_{2}^{a}\right) \\
& \bar{D}=\bar{\partial}+\bar{A}=\frac{1}{2}\left(\partial_{1}-i \partial_{2}\right)+\frac{1}{2}\left(A_{1}-i A_{2}\right)=\frac{1}{2}\left(\partial_{1}-i \partial_{2}\right)+\left(-i t_{a}\right) \frac{1}{2}\left(A_{1}^{a}-i A_{2}^{a}\right)
\end{aligned}
$$

One can parametrize the gauge field in terms of a complex matrix $M$ as $A=-\partial M M^{-1}$, $\bar{A}=M^{\dagger-1} \bar{\partial} M^{\dagger}$, which yields the second expression in (1) in terms of the WZW action with $H=M^{\dagger} M$. In (2), $\left\{t_{a}\right\}$ are a set of hermitian matrices forming a basis for the Lie algebra of the gauge group, $A_{R}=1$ if the fields are in the fundamental representation $(\mathrm{F})$, otherwise, for representation $R$, it is defined by $\operatorname{Tr}\left(t_{a} t_{b}\right)_{R}=A_{R} \operatorname{Tr}\left(t_{a} t_{b}\right)_{F}$. The integration is over the two-dimensional space $\mathcal{M}$ of interest; in the second term of $S_{\mathrm{WZW}}$ we extend the fields to a three-manifold $\mathcal{M}^{3}$ whose boundary is $\mathcal{M}$, as usual.

The WZW action we have written can also be expressed directly in terms of the gauge potentials, which is useful for explicit computations in a gauge theory. It reads

$$
\begin{aligned}
S_{\mathrm{WZW}}(H) & =\frac{1}{\pi}\left[\int d^{2} z \operatorname{Tr}(A \bar{A})-\pi \mathcal{I}(A)-\pi \overline{\mathcal{I}}(\bar{A})\right] \\
\mathcal{I}(A) & =\sum_{2}^{\infty} \frac{(-1)^{n}}{n \pi^{n}} \int d^{2} z_{1} \cdots d^{2} z_{n} \frac{\operatorname{Tr}\left(A\left(z_{1}, \bar{z}_{1}\right) A\left(z_{2}, \bar{z}_{2}\right) \cdots A\left(z_{n}, \bar{z}_{n}\right)\right)}{\left(\bar{z}_{1}-\bar{z}_{2}\right)\left(\bar{z}_{2}-\bar{z}_{3}\right) \cdots\left(\bar{z}_{n}-\bar{z}_{1}\right)}
\end{aligned}
$$

and $\overline{\mathcal{I}}(\bar{A})$ is similar with $A \rightarrow \bar{A}, \bar{z}_{1}-\bar{z}_{2} \rightarrow z_{1}-z_{2}$, etc. for the terms in the denominator of the expression in (3).

The fact that we have a complex structure for $\mathbb{R}^{2}$ is important in constructing this mass term. The WZW action, and hence the mass term, can be written for any Riemann surface, viewed as a complex manifold, by a simple generalization as

$$
\begin{aligned}
S_{\mathrm{WZW}}(H)=\frac{1}{8 \pi} & \int_{\mathcal{M}} d^{2} x \sqrt{g} g^{a b} \operatorname{Tr}\left(\partial_{a} H \partial_{b} H^{-1}\right) \\
& +\frac{i}{12 \pi} \int_{\mathcal{M}^{3}} \operatorname{Tr}\left(H^{-1} d H \wedge H^{-1} d H \wedge H^{-1} d H\right)
\end{aligned}
$$


where $g_{a b}$ is the metric tensor for the two-manifold.

Consider now the extension of this to four dimensions. A mass term, similar to (1), can be written down if we can identify two complex coordinates out of the four real coordinates of $\mathbb{R}^{4}$. But there are many choices for a complex (or even a Kähler) structure. We can understand the inequivalent choices as follows. If we choose one set of complex combinations, say $\omega_{1}=x_{0}+i x_{3}, \omega_{2}=x_{2}-i x_{1}$, then a $U(2)$ transformation of $\left(\omega_{1}, \omega_{2}\right)$ does not change the complex structure. In particular, a holomorphic function of $\omega=\left(\omega_{1}, \omega_{2}\right)$ remains holomorphic under the $U(2)$ transformation. However, we can do a rotation of all four coordinates, as $x_{\mu} \rightarrow x_{\mu}^{\prime}=R_{\mu}^{\nu} x_{\nu}$, where $R_{\mu}^{\nu}$ is a rotation matrix, and this does lead to a different structure given by $x_{0}^{\prime}+i x_{3}^{\prime}, x_{2}^{\prime}-i x_{1}^{\prime}$. So the inequivalent ways of combining $\left(x_{0}, x_{1}, x_{2}, x_{3}\right)$ into complex combinations are parametrized by $S O(4) / U(2) \sim S^{2}$. More explicitly, introduce a two-spinor $\left(\pi_{1}, \pi_{2}\right)$ with the identification $\pi=\left(\pi_{1}, \pi_{2}\right) \sim \lambda\left(\pi_{1}, \pi_{2}\right), \lambda \in \mathbb{C}-\{0\}$. The $\pi$ 's parametrize $\mathbb{C P}^{1} \sim S^{2}$. The complex combinations can be taken as

$$
\left(\begin{array}{l}
\omega_{1} \\
\omega_{2}
\end{array}\right)=\left(x_{0}-i \sigma_{i} x_{i}\right) \pi=\left[\begin{array}{cc}
x_{0}-i x_{3} & -x_{2}-i x_{1} \\
x_{2}-i x_{1} & x_{0}+i x_{3}
\end{array}\right]\left(\begin{array}{l}
\pi_{1} \\
\pi_{2}
\end{array}\right)
$$

where $\sigma_{i}$ are the Pauli matrices. We can also define a real unit vector $Q_{i}=\bar{\pi} \sigma_{i} \pi /(\bar{\pi} \pi)$. We then find

$$
(\bar{\pi} \omega, \bar{\omega} \pi)=\left(x_{0}-i \vec{Q} \cdot \vec{x}, x_{0}+i \vec{Q} \cdot \vec{x}\right) \equiv(z, \bar{z})
$$

These constitute two of the complex coordinates. The remaining two transverse coordinates are given by $\vec{x} \times \vec{Q}$. The unit vector $Q_{i}$ gives an alternate way to parametrize $S^{2}$. For any fixed choice of $\vec{Q}$, we do lose rotational invariance but we can construct an invariant mass term as [5]

$$
\Gamma=-k \int d \mu_{S^{2}} d^{2} x^{T} S_{\mathrm{WZW}}(H)
$$

where $H=M^{\dagger} M$ and

$$
\begin{aligned}
A & =\frac{1}{2}\left(A_{0}+i \vec{Q} \cdot \vec{A}\right)=-\frac{1}{2}\left(\partial_{0}+i \vec{Q} \cdot \nabla\right) M M^{-1} \\
\bar{A} & =\frac{1}{2}\left(A_{0}-i \vec{Q} \cdot \vec{A}\right)=\frac{1}{2} M^{\dagger-1}\left(\partial_{0}-i \vec{Q} \cdot \nabla\right) M^{\dagger}
\end{aligned}
$$

The integrations over all orientations of $\vec{Q}$, signified in (8) by $d \mu_{S^{2}}$, and over the transverse coordinates $x^{T}$, will make this mass term rotationally invariant. The integration over $z, \bar{z}$ is part of $S_{\mathrm{WZW}}(H)$, so the final result in (7) will have integration over all four coordinates with the measure $d^{4} x$. This mass term is also obviously gauge-invariant, in the same way as in two dimensions.

A number of comments are in order at this point. First of all, the key idea here is to use a pair of complex coordinates or more generally a two-dimensional complex subspace to construct the WZW action. This necessarily entails a lack of rotational symmetry. Symmetry is restored by integrating over all possible choices of complex coordinates. 
In other words, we may think of the total space of interest as the bundle of complex structures on $\mathbb{R}^{4}$. This is basically the way twistor space is defined [11]. In the present case, where the base space is flat, the bundle is trivial. One could do an analogous construction for other spaces, a notable example being $S^{4}$. For this latter case, we cannot have a global complex structure, so combinations of coordinates into complex ones correspond to local complex structures and one is considering the bundle of local complex structures over $S^{4}$. The bundle space is then $\mathbb{C P}^{3}$, with $S^{2}$ as the fiber and $S^{4}$ as the base, and the bundle is topologically nontrivial. In any case, the key point here is to consider the bundle of local complex structures, trivial or nontrivial.

Secondly, while the twistor space genesis of (7) may be mathematically gratifying, one may ask whether this mass term has anything to do with physics. The remarkable fact is that it does. Of course, its use in physics needs a continuation to Minkowski signature, not the Euclidean one we have used so far. This continuation can be done by the rules

$$
\begin{aligned}
& A \rightarrow \frac{1}{2}\left(A_{0}+\vec{Q} \cdot \vec{A}\right)=-\frac{1}{2}\left(\partial_{0}+\vec{Q} \cdot \nabla\right) M M^{-1} \\
& \bar{A} \rightarrow \frac{1}{2}\left(A_{0}-\vec{Q} \cdot \vec{A}\right)=\frac{1}{2} M^{\dagger-1}\left(\partial_{0}-\vec{Q} \cdot \nabla\right) M^{\dagger}
\end{aligned}
$$

If we consider a physical system described by a nonabelian gauge theory such as quantum chromodynamics (QCD), then, at finite temperature where we get a plasma of gluons (and quarks if they are included), the terms in the standard perturbative expansion have infrared divergences. There is an infinite sequence of terms, which are the leading infrared divergent terms, known as hard thermal loops (HTL) [12]. These are special to the case of nonzero temperature and are in addition to the usual divergences (both in the ultraviolet and infrared) in the theory at zero temperature. These HTL terms have to be summed up and included at the lowest order to reformulate perturbation theory without infrared divergences. (This has to be done in a self-consistent way, the technology for this is well understood.) The summation of the HTL terms is a screening effect for the electric-type forces corresponding to the nonabelian gauge field. In fact it is the nonabelian generalization of the Debye screening effect well known for the Abelian plasma (and electrolytes). The sum of the HTL terms can be interpreted as a mass term, primarily for the $A_{0}$-component of the gauge field, with some contributions to the other components as well to satisfy the Gauss law. The HTL contributions can be calculated in the field theory at finite temperature and the result of the detailed calculations at finite temperature is exactly the mass term (7), with the continuation in (9), and with $k=\left(N+\frac{1}{2} N_{F}\right) T^{2} / 6$ [5]. This value of $k$ is for the case of an $S U(N)$ gauge theory, with $N_{F}$ massless fermion flavors and $T$ denotes the temperature of the plasma. Thus, quite remarkably, what was defined purely as a mathematical generalization is indeed realized in explicit calculations in a very physical context, namely, the quark-gluon plasma.

A similar screening effect also occurs for a degenerate gas of quarks with a nonzero 
total baryon number, such as can occur deep inside a neutron star. This is the nonabelian generalization of the well-known Thomas-Fermi screening effect for electron gases. The mass term describing this is again of the same form, with $k=\mu_{q}^{2} / 4 \pi^{2}$, where $\mu_{q}$ is the chemical potential for the quark number ( $=\frac{1}{3}$ of the baryon number) [13].

Finally, we may raise the question of Lorentz invariance. The Minkowski continuation of the mass term as written in (7) is not Lorentz-invariant. Physically, this is indeed as it should be, since thermal equilibrium and the specification of the temperature are obtained in the rest frame of the plasma without any overall drift velocity. For a Lorentzinvariant result, we need one more parameter, the overall drift velocity of the plasma, whose Lorentz transformation will lead to an invariant result. The relevant form of the mass term (i.e., the generalization for the moving plasma) was worked out many years ago and takes the form [14]

$$
\begin{aligned}
\Gamma & =-k \int d \mu d^{2} x^{T} S_{W Z W}(H) \\
d \mu & =2 i \frac{\pi \cdot d \pi \bar{\pi} \cdot d \bar{\pi} \xi \cdot d \xi \bar{\xi} \cdot d \bar{\xi}}{(\pi \cdot \xi)^{2}(\bar{\pi} \cdot \bar{\xi})^{2}} \delta[\xi(e \cdot p) \bar{\pi}] \delta[\pi(e \cdot p) \bar{\xi}]
\end{aligned}
$$

We have introduced two sets of two-component $S L(2, \mathbb{C})$ spinors, $\pi^{A}, \xi^{A}, A=1,2$, with $\pi^{\dot{A}}=\overline{\pi^{A}}$ and $\xi^{\dot{A}}=\overline{\xi^{A}}$ as their complex conjugates. The components of the gauge fields used to define $M$ and $M^{\dagger}$, and hence $H$, are given by

$$
A_{\pi}=\frac{1}{2} \pi(e \cdot A) \bar{\pi}, \quad A_{\xi}=\frac{1}{2} \xi(e \cdot A) \bar{\xi}
$$

Further $e^{\mu}=\left(\mathbf{1}, \sigma^{i}\right)$, and $p_{\mu}$ in (10) denotes the drift 4-velocity of the plasma. The derivatives are defined in a way similar to the $A$ 's given above, namely by (11) with $A \rightarrow \partial$, with corresponding expressions for the coordinates. Notice the presence of the $\delta$-functions in $d \mu$. Upon integration, they enforce a relation between the two spinors in a way which depends on $p^{\mu}$. In the rest frame of the plasma, with $p^{\mu}=(1,0,0,0)$, expression (10) reproduces the previous result (7).

Another feature worthy of remark is that the combinations of the gauge potentials in (9), as well as derivatives, can be written as $n \cdot A, n \cdot \partial$ and $n^{\prime} \cdot A, n^{\prime} \cdot \partial$, where $n^{\mu}=$ $\left(1, i Q_{i}\right) n^{\prime \mu}=\left(1,-i Q_{i}\right)=\bar{n}^{\mu}$ in Euclidean space. These are complex null vectors. Upon continuation to Minkowski space, we get $n^{\mu}=\left(1, Q_{i}\right) n^{\prime \mu}=\left(1,-Q_{i}\right)$, which are real null vectors. Thus the bundle space we are considering is the bundle of lightcones over Minkowski space [11].

Turning now to three-dimensional space, obviously we cannot combine coordinates pairwise into complex combinations, so an immediate generalization seems difficult. However, there is a mathematical structure known as the Sasakian which can exist on certain odd-dimensional manifolds and which has been suggested as the closest we can get to a Kähler structure. We can try to utilize this to construct a mass term. In the next 
section, we give a general discussion of Sasakians for $S^{3}$ and $\mathbb{R}^{3}$ and write down this mass term. The final result agrees with what was suggested as the magnetic screening mass for the gluon plasma many years ago, although the Sasakian connection was not apparent at that time.

\section{$3 S^{3}$ and $\mathbb{R}^{3}$ as Sasakian manifolds and a 3d mass term}

We begin by briefly recalling the definition of a Sasakian manifold [15]. Let $\mathcal{M}$ be an odddimensional Riemannian manifold with the metric $d s_{\mathcal{M}}^{2}$. The Riemannian cone for $\mathcal{M}$ is $\mathcal{M} \times \mathbb{R}_{+}$with the cone metric

$$
d s^{2}=d r^{2}+r^{2} d s_{\mathcal{M}}^{2}
$$

where $r \in \mathbb{R}_{+}$is the additional coordinate along the $\mathbb{R}_{+}$direction. The manifold $\mathcal{M}$ is said to be a contact manifold if there is a one-form $\hat{\Theta}$ on $\mathcal{M}$ such that the two-form

$$
\Omega=r^{2} d \hat{\Theta}+2 r d r \hat{\Theta}
$$

is symplectic. The manifold $\mathcal{M}$ endowed with $\hat{\Theta}$ is Sasakian if the two-form $\Omega$ and the metric $d s^{2}$ on the cone, i.e., (12), are Kähler. Since $\mathcal{M}$ is a transverse cross-section of the cone, it inherits many properties from the Kähler structure of the cone. In fact, it is generally considered that the Sasakian structure is the closest one can get to Kähler-type properties for an odd-dimensional space.

We can apply this specifically to $S^{3}$ by considering its embedding in $\mathbb{R}^{4}$ and taking $r$ as the radial coordinate. Removing the origin, $\mathbb{R}^{4}-\{0\}$ has the cone structure, with the metric on the cone being the flat Euclidean metric $d s^{2}=d x_{0}^{2}+d x_{1}^{2}+d x_{2}^{2}+d x_{3}^{2}$. To identify $S^{3}$ as a Sasakian space, we need to write this metric as a Kähler metric. As discussed in the last section, there are an infinity of inequivalent ways of doing this, the possible complex combinations being parametrized by $\pi$ which form the homogeneous coordinates for $\mathbb{C P}^{1}$. Using the freedom of scaling, $\pi \sim l \pi, l \in \mathbb{C}-\{0\}$, we can bring it to the form

$$
\begin{aligned}
\left(\begin{array}{l}
\pi_{1} \\
\pi_{2}
\end{array}\right) & =\left(\begin{array}{l}
-e^{-i \frac{\varphi}{2}} \cos \frac{\theta}{2} \\
-e^{i \frac{\varphi}{2}} \sin \frac{\theta}{2}
\end{array}\right) \\
& =\left[\begin{array}{cc}
-e^{-i \frac{\varphi}{2}} \sin \frac{\theta}{2} & -e^{-i \frac{\varphi}{2}} \cos \frac{\theta}{2} \\
e^{i \frac{\varphi}{2}} \cos \frac{\theta}{2} & -e^{i \frac{\varphi}{2}} \sin \frac{\theta}{2}
\end{array}\right]\left(\begin{array}{l}
0 \\
1
\end{array}\right) \equiv g\left(\begin{array}{l}
0 \\
1
\end{array}\right)
\end{aligned}
$$

The complex combinations for $\mathbb{R}^{4}$ can be taken as in (5). But as mentioned earlier, we are free to do a $U(2)$ rotation of the complex combinations without changing the complex structure. For our purpose here, it is useful to do this using $g^{\dagger}$, thus defining

$$
\omega=g^{\dagger}\left(x_{0}-i \vec{\sigma} \cdot \vec{x}\right) \pi=g^{\dagger}\left(x_{0}-i \vec{\sigma} \cdot \vec{x}\right) g\left(\begin{array}{l}
0 \\
1
\end{array}\right)
$$




$$
=\left(x_{0}-i \sigma_{k} R_{k i} x_{i}\right)\left(\begin{array}{l}
0 \\
1
\end{array}\right)
$$

For the choice of $\pi$ in (14), the components of the orthogonal matrix $R_{k i}$ are given by

$$
R_{k i}=\left[\begin{array}{ccc}
-\cos \theta \cos \varphi & -\cos \theta \sin \varphi & \sin \theta \\
-\sin \varphi & \cos \varphi & 0 \\
-\sin \theta \cos \varphi & -\sin \theta \sin \varphi & -\cos \theta
\end{array}\right]
$$

In terms of the complex coordinates $\omega$, the Kähler forms and metric can be taken as

$$
\begin{aligned}
\Omega & =i\left(d \bar{\omega}_{1} \wedge d \omega_{1}+d \bar{\omega}_{2} \wedge d \omega_{2}\right)=d \mathcal{A} \\
\mathcal{A} & =\frac{i}{2}\left[\bar{\omega}_{1} \wedge d \omega_{1}-\omega_{1} \wedge d \bar{\omega}_{1}+\bar{\omega}_{2} \wedge d \omega_{2}-\omega_{2} \wedge d \bar{\omega}_{2}\right] \\
d s^{2} & =d \bar{\omega}_{1} d \omega_{1}+d \bar{\omega}_{2} d \omega_{2}
\end{aligned}
$$

Using $\omega_{1}, \omega_{2}$ from (15), we can now separate out the radial coordinate, writing

$$
\begin{aligned}
& \omega_{1}=-i\left(R_{1 i}-i R_{2 i}\right) x_{i}=r\left[-i\left(R_{1 i}-i R_{2 i}\right) \phi_{i}\right] \\
& \omega_{2}=x_{0}+i R_{3 i} x_{i}=r\left[\phi_{0}+i R_{3 i} \phi_{i}\right]
\end{aligned}
$$

with $\phi_{0} \phi_{0}+\phi_{i} \phi_{i}=1$. It is straightforward to simplify $\mathcal{A}$ to get $\mathcal{A}=r^{2} \hat{\Theta}$, with

$$
\begin{aligned}
\hat{\Theta} & =R_{3 i}\left(\phi_{i} d \phi_{0}-\phi_{0} d \phi_{i}\right)+\frac{i}{2}[(n \cdot \phi) d(\bar{n} \cdot \phi)-(\bar{n} \cdot \phi) d(n \cdot \phi)] \\
n_{i} & =R_{1 i}+i R_{2 i}=(-\cos \theta \cos \varphi-i \sin \varphi,-\cos \theta \sin \varphi+i \cos \varphi, \sin \theta)
\end{aligned}
$$

We have chosen a Kähler metric for the cone and we see that $\Omega=d \mathcal{A}$ does have the required structure (13). This is basically the Sasakian structure on $S^{3}$. Notice that the second term in $\hat{\Theta}$, namely,

$$
\alpha=\frac{i}{2}[(n \cdot \phi) d(\bar{n} \cdot \phi)-(\bar{n} \cdot \phi) d(n \cdot \phi)]
$$

defines a local Kähler structure for the two-dimensional subspace transverse to $R_{3 i} \phi_{i}$. This is only local, since the separation of the third direction on $S^{3}$ can only be local. The existence of such a local transverse Kähler structure is a feature of Sasakian manifolds.

The vectors $n_{i}, \bar{n}_{i}$ define the choice of complex combinations on the cone. ( $R_{3 i}$ is not independent, it is proportional to $(\vec{n} \times \vec{n})_{i}$. ) A particular Kähler structure on the cone corresponds to a particular choice of these vectors, each of them leading to a particular Sasakian structure for $S^{3}$. We have an $S^{2}$ worth of such choices, so the total space we are considering is the bundle of Sasakians over $S^{3}$. In this sense, it is the natural equivalent in three dimensions of the twistor space in four dimensions 1 . Notice that, since $R_{k i}$ is an orthogonal matrix,

$$
n_{i} n_{i}=\bar{n}_{i} \bar{n}_{i}=0, \quad n_{i} \bar{n}_{i}=2
$$

\footnotetext{
${ }^{1}$ In Ref. [15], Boyer and Galicki study a particular version of what they name as the twistor space for Sasakian manifolds. They also mention that there could be another object which deserves the name of twistor space for Sasakians. The latter one is the trivial $S^{2}$ bundle for a Sasakian manifold inheriting the structure from the Kähler structure on the cone. It is this latter definition which applies to our case here.
} 
In other words, $n_{i}, \bar{n}_{i}$ are complex null vectors, normalized by the second relation in (21). Thus we may think of the bundle of Sasakians of $S^{3}$ as the bundle of complex null rays. In this case, the bundle is still trivial just as it was in $\mathbb{R}^{4}$.

To proceed further, we introduce stereographic coordinates $y_{i}$ for $S^{3}$ by

$$
\phi_{0}=\frac{y^{2}-R^{2}}{y^{2}+R^{2}}, \quad \phi_{i}=\frac{2 y_{i} R}{y^{2}+R^{2}}
$$

We also introduce the notation $z=n \cdot y, \bar{z}=\bar{n} \cdot y, R_{3 i} y_{i}=v$. The Kähler potential for the transverse local Kähler one-form $\alpha$ in (20) is

$$
K_{T}=(n \cdot \phi)(\bar{n} \cdot \phi)=\frac{4 R^{2} \bar{z} z}{\left(\bar{z} z+v^{2}+R^{2}\right)^{2}}
$$

If we take the large $R$ limit, which corresponds to blowing up the $S^{3}$ to get $\mathbb{R}^{3}$, we find

$$
\begin{aligned}
\hat{\Theta} & \approx \frac{2 d v}{R}+\frac{2 i}{R^{2}}(z d \bar{z}-\bar{z} d z) \\
K_{T} & \approx 4 \frac{\bar{z} z}{R^{2}}
\end{aligned}
$$

This will be useful in applying our results to gauge fields in $\mathbb{R}^{3}$.

We can now write down the WZW action for the transverse space with the coordinates $z, \bar{z}$, with the (local) Kähler metric $\left(\partial \bar{\partial} K_{T}\right) d z d \bar{z}$. The factors involving $\left(\partial \bar{\partial} K_{T}\right)$ will drop out of $S_{\mathrm{WZW}}$ because of its conformal invariance. The action is thus the integral of a differential two-form given as

$$
\begin{aligned}
S_{\mathrm{WZW}}(H)= & -\frac{i}{4 \pi} \int_{\mathcal{M}} \operatorname{Tr}\left(\partial H \wedge \bar{\partial} H^{-1}\right)+\frac{i}{12 \pi} \int_{\mathcal{M}^{3}} \operatorname{Tr}\left(H^{-1} d H \wedge H^{-1} d H \wedge H^{-1} d H\right) \\
= & -\frac{i}{4 \pi}\left[\int_{\mathcal{M}} \operatorname{Tr}\left(\partial H \wedge \bar{\partial} H^{-1}\right)\right. \\
& \left.-\int_{\mathcal{M}^{3}} \operatorname{Tr}\left[H^{-1} d H \wedge\left(H^{-1} \partial H \wedge H^{-1} \bar{\partial} H-H^{-1} \bar{\partial} H \wedge H^{-1} \partial H\right)\right]\right]
\end{aligned}
$$

The mass term of interest can now be written down as $\Gamma=m^{2} S_{m}$, with [14]

$$
\begin{aligned}
S_{m}= & -\int d \mu\left(S^{2}\right) \hat{\Theta} S_{\mathrm{WZW}}(H) \\
= & \frac{i}{4 \pi} \int d \mu\left(S^{2}\right) \hat{\Theta} \wedge\left[\operatorname{Tr}\left(\partial H \wedge \bar{\partial} H^{-1}\right)\right. \\
& \left.\quad-\operatorname{Tr}\left[H^{-1} d H \wedge\left(H^{-1} \partial H \wedge H^{-1} \bar{\partial} H-H^{-1} \bar{\partial} H \wedge H^{-1} \partial H\right)\right]\right]
\end{aligned}
$$

The same expression also applies to the large $R\left(\right.$ or $\mathbb{R}^{3}$ ) limit, where the one-form $\hat{\Theta}$ and the potential $K_{T}$ simplify as given in (24). 
The expression (26) may still seem rather cryptic, but it is straightforward to work out the expression as a series in terms of the gauge potentials, after Fourier transforming to momentum space. The first two terms are [16]

$$
\begin{aligned}
& S_{m}= \frac{1}{2} \int \frac{d^{3} k}{(2 \pi)^{3}} A_{i}^{a}(-k) A_{j}^{a}(k)\left(\delta_{i j}-\frac{k_{i} k_{j}}{\vec{k}^{2}}\right) \\
&+\int \frac{d^{3} k}{(2 \pi)^{3}} \frac{d^{3} q}{(2 \pi)^{3}} A_{i}^{a}(k) A_{j}^{b}(q) A_{k}^{c}(-k-q) f^{a b c} V_{i j k}(k, q,-k-q) \\
& V_{i j k}(k, q,-(k+q))= \frac{i}{6}\left[\frac{1}{k^{2} q^{2}-(q \cdot k)^{2}}\right]\left[\left\{\frac{q \cdot k}{k^{2}}-\frac{q \cdot(q+k)}{(q+k)^{2}}\right\} k_{i} k_{j} k_{k}\right. \\
&\left.+\frac{k \cdot(q+k)}{(q+k)^{2}}\left(q_{i} q_{j} k_{k}+q_{k} q_{i} k_{j}+q_{j} q_{k} k_{i}\right)-(q \leftrightarrow k)\right]
\end{aligned}
$$

\section{Properties of the 3d mass term}

Our arguments in arriving at (26) show that it has a deep and interesting mathematical structure and that it is the most natural generalization to three dimensions of the results in two and four dimensions. But we can again ask the crucial question of whether it has anything to do with physics. Indeed that is the case, the motivation from physics is what led to this mass term, for $\mathbb{R}^{3}$, many years ago, although the Sasakian structure was not clear at that time [14]. The general expectation is that in nonabelian gauge theories a mass gap will be dynamically generated, so potentially, one can get a term like (26) in the effective action for such theories. This will be a highly nonperturbative result. One can attempt to demonstrate this, and calculate the coefficient $m^{2}$, via a gap equation approach where we add and subtract the same term to the standard Yang-Mills action,

$$
\begin{aligned}
S & =S_{\mathrm{YM}}+m^{2} S_{m}-\Delta S_{m}=\tilde{S}-\Delta S_{m} \\
\tilde{S} & =S_{\mathrm{YM}}+m^{2} S_{m}
\end{aligned}
$$

The idea is to consider $m^{2}$ as the exact value of the mass generated by interactions while $\Delta$ is taken to have a loop expansion of the form $\Delta=\Delta^{(1)}+\Delta^{(2)}+\ldots$. Calculations can be done in a loop expansion, with the action $\tilde{S}$ used to construct the propagators and vertices at the tree level, but $\Delta$ starts at the one-loop level. Since $m^{2}$ is taken to be the exact dynamically generated mass, the pole of the propagator must remain at $k_{0}^{2}-\vec{k}^{2}=m^{2}$ as loop corrections are added. This requires choosing $\Delta^{(1)}$ to cancel the one-loop shift of the pole, $\Delta^{(2)}$ to cancel the two-loop shift of the pole, etc., as is usually done for mass renormalization. After this is done, the $\Delta$ so obtained must still equal $\mathrm{m}^{2}$, since the theory is defined by just the YM action. Thus we must impose the condition

$$
\Delta=\Delta^{(1)}+\Delta^{(2)}+\ldots=m^{2}
$$


This statement of equating the corrections to the mass term to $\mathrm{m}^{2}$ is the gap equation which determines $m^{2}[16]$. (This strategy can be continued to arbitrary orders of calculation [7, 16].) Notice that the approach is completely gauge-invariant. The calculation of $m^{2}$ along these lines was carried out in Ref.[16] and gave the value $m \approx 1.19\left(e^{2} c_{A} / 2 \pi\right)$, where $e$ is the gauge coupling and $c_{A}$ is the quadratic Casimir value for the adjoint representation of the gauge group. (For other related approaches to the magnetic screening mass, see Refs.[17], [7].)

The gap equation can be viewed as the result of the summation of an infinite number of Feynman diagrams, a particular sequence being chosen by the form of the mass term. A very different approach is to use the Schrödinger equation in the Hamiltonian formulation of the theory and to solve it for the ground state wave functional in a low energy approximation. Such an approach, which has been developed in a series of articles [18], leads to a prediction for the string tension (which is in good agreement with lattice simulations [19]) and a value for $m$ as $e^{2} c_{A} / 2 \pi$. This is close to the value obtained from the gap equation analysis. Yet another independent validation of the result comes from using the same Hamiltonian approach to calculate the Casimir energy for two parallel plates [20]. One can then obtain a direct and independent numerical estimate of the value of the mass by a lattice simulation of the parallel plate geometry for the Yang-Mills theory. Such a simulation yields the same value of $e^{2} c_{A} / 2 \pi$ to within a fraction of a percent [21].

There are two other observations regarding this mass term which might be interesting. The first is about the one-loop correction generated by the mass term which determines the gap equation. Let us denote, in any gauge theory, the correction to the two-point function as $\int A_{i}^{a}(-k) \Pi_{i j}(k) A_{j}^{a}(k)$. Then one can analyze some of the excitations which can occur in intermediate states of the one-loop graph, corresponding to a unitarity cut of the diagram, by the singularity structure of $\Pi_{i j}(k)$. As an example, if we use a mass term

$$
S_{m}=\int d^{3} x \operatorname{Tr}\left[F_{i}\left(\frac{1}{D^{2}}\right) F_{i}\right], \quad F_{i}=\frac{1}{2} \epsilon_{i j k} F_{j k},
$$

one can show that there are singularities at $k^{2}=0$ in $\Pi_{i j}(k)$ indicating that there are still zero-mass excitations present in the spectrum [7]. One way to understand this is to note that if we write (30) in terms of local monomials with an auxiliary field, we get

$$
S_{m}=-\int d^{3} x \operatorname{Tr}\left[\frac{1}{2} \phi_{i}\left(-D^{2}\right) \phi_{i}+\phi_{i} F_{i}\right]
$$

This would give propagating massless solutions corresponding to $\square \phi_{i}=0$ in the absence of $A_{i}$. In contrast to this, $\Pi_{i j}(k)$ resulting from the mass term (26) or (27) has no zero-mass threshold singularities, at least to the one-loop order the calculations have been carried out. One way to understand this may be to note that the mass term rewrit- 
ten in terms of local monomials with auxiliary fields is

$$
\begin{aligned}
S_{m}= & \int d \mu\left(S^{2}\right)\left[\int d x^{T} S_{\mathrm{WZW}}(G)\right. \\
& \left.+\frac{1}{\pi} \int d^{3} x \operatorname{Tr}\left(G^{-1} \bar{\partial} G A-\bar{A} \partial G G^{-1}+A G^{-1} \bar{A} G-A \bar{A}\right)\right]
\end{aligned}
$$

The equations of motion for the group element $G$ leads to the solution

$$
G=M^{\dagger-1} \tilde{V}(z) V(\bar{z}) M^{-1}
$$

Since the matrices $M, M^{\dagger}$ are only defined up to the ambiguity $M \rightarrow M V^{-1}(\bar{z}), M^{\dagger} \rightarrow$ $\tilde{V}(z) M^{\dagger}$ by the equations $A=-\partial M M^{-1}, \bar{A}=M^{\dagger-1} \bar{\partial} M^{\dagger}$, we can absorb $\tilde{V}(z) V(\bar{z})$ in (33) into the definition of $M, M^{\dagger}$. Thus there are no independent solutions, or independent degrees of freedom, for the auxiliary field.

Our second observation is about the use of this mass term in the context of the quarkgluon plasma. The hard thermal loops generate a screening mass (7) or (10) for the chromoelectric forces, the mass term (26) can describe the magnetic screening or the magnetic mass of the plasma. In carrying out calculations at finite temperature, one can see that, even after taking account of the hard thermal loops and the corresponding chormoelectric screening effects, there are still infrared divergences left over in the nonabelian theory. These are cured by the screening mass for the chromomagnetic interactions, so the dynamical generation of such a mass term is an important feature. At very high temperatures, the $3+1$ dimensional theory can be approximated by the same theory in three Euclidean dimensions, i.e., there is a dimensional reduction, the coupling of the $3 \mathrm{~d}$ theory being $e^{2}=g^{2} T$, where $g$ is the $4 \mathrm{~d}$ coupling. The dynamically generated mass of the $3 \mathrm{~d}$ theory can thus be interpreted as the magnetic screening mass of the high temperature limit of the $4 \mathrm{~d}$ theory [22]. For this idea to be implemented in the full four-dimensional theory, we again need a $4 \mathrm{~d}$-Lorentz invariant form of the mass term. It is indeed possible

to construct such an invariant mass term [14]. The result is exactly of the form given in (10) with one change. Instead of the components of $A_{\mu}$ given (11), we must use

$$
A_{\pi}=\frac{1}{2} \pi(e \cdot A) \bar{\xi}, \quad A_{\xi}=\frac{1}{2} \xi(e \cdot A) \bar{\pi}
$$

Notice that in each of these combinations there is mixing of the spinors $\pi, \xi$, unlike the situation in (11).

\section{Acknowledgements}

I thank A.P. Balachandran and Denjoe O'Connor for discussions on the Sasakian structure. This research was supported in part by the U.S. National Science Foundation grant PHY-1820721 and by PSC-CUNY awards. 


\section{References}

[1] R. Jackiw and S-Y. Pi, Phys. Rev. Lett. 64, 2969 (1990).

[2] R. Jackiw and V.P. Nair, Phys. Rev. D43, 1933 (1991); Phys. Rev. Lett. 73, 2007 (1994); Phys. Lett. B480, 237 (2000); Phys. Lett. B551, 166 (2003).

[3] R. Jackiw, V.P. Nair and S-Y. Pi, Phys. Rev. D62, 085018 (2000).

[4] B. Bistrovic et al, Phys. Rev. D67, 025013 (2003); R. Jackiw, V.P. Nair, S-Y. Pi and A.P. Polychronakos, J. Phys. A: Math. Gen. 37, R327 (2004).

[5] R. Efraty and V.P. Nair, Phys. Rev. Lett. 68, 2891 (1992); Phys. Rev. D47, 5601 (1993).

[6] R. Jackiw and V.P. Nair, Phys. Rev. D48, 4991 (1993).

[7] R. Jackiw and So-Young Pi, Phys. Lett. B368, 131 (1996); ibid. B403, 297 (1997).

[8] E. Witten, Commun. Math. Phys., 92, 455 (1984); S.P. Novikov, Usp. Mat. Nauk 37, 3 (1982).

[9] A.M. Polyakov and P.B. Wiegmann, Phys. Lett. B141, 223 (1984).

[10] J. S. Schwinger, Phys. Rev. 128, 2425 (1962).

[11] R. Penrose, J. Math. Phys. 8, 345 (1967). The subject is by now very well developed, see R. Penrose and M.A.H. MacCallum, Phys. Rep. 6, 241 (1972); R. Penrose and W. Rindler, Spinors and Spacetime, 2 volumes (Cambridge University Press, 1984 \& 1987); S.A. Hugget and K.P. Tod, An Introduction to Twistor Theory (Cambridge University Press, 1993).

[12] R. Pisarski, Physica A158, 246 (1989); Phys. Rev. Lett. 63, 1129 (1989); E. Braaten and R. Pisarski, Phys. Rev. D42, 2156 (1990); Nucl. Phys. B337, 569 (1990); ibid. B339, 310 (1990); Phys. Rev. D45, 1827 (1992); J. Frenkel and J.C. Taylor, Nucl. Phys. B334, 199 (1990); J.C. Taylor and S.M.H. Wong, Nucl. Phys. B346, 115 (1990).

[13] G. Alexanian and V.P. Nair, Phys. Lett. B390, 370 (1997).

[14] V.P. Nair, Phys. Lett. B352, 117 (1995).

[15] S. Sasaki, Tohoku Math. J. 2, 459 (1960); For further developments and reviews, see C. Boyer and K. Galicki, Surveys Diff. Geom. 7, 123 (1999) |arXiv:hep-th/9810250|.

[16] G. Alexanian and V.P. Nair, Phys. Lett. B352, 435 (1995). 
[17] J.M. Cornwall, Phys. Rev. D10, 500 (1974); ibid. D26, 1453 (1982); ibid. D57, 3694 (1998); W. Buchmuller and O. Philipsen, Nucl. Phys. B443 (1995) 47; O. Philipsen, in TFT-98: Thermal Field Theories and their Applications, U. Heinz (ed.), hep-ph/9811469 J. M. Cornwall and B. Yan, Phys. Rev. D53, 4638 (1996); J.M. Cornwall, Phys. Rev. D76, 025012 (2007).

[18] D. Karabali and V.P. Nair, Nucl. Phys. B464, 135 (1996); Phys. Lett. B379, 141 (1996); D. Karabali, Chanju Kim and V.P. Nair, Nucl. Phys. B524, 661 (1998); Phys. Lett. B434, 103 (1998); D. Karabali, V.P. Nair and A. Yelnikov, Nucl. Phys. B824, 387 (2010); For a short review, see V.P. Nair, arXiv:0910.3252, Proceedings of Science, POS(QCD-TNT09) 030, http://pos.sissa.it//archive/conferences/087/030/QCD-TNT09_030.pdf.

[19] M. Teper, Phys. Rev. D59, 014512 (1999) and references therein; B. Lucini and M. Teper, Phys. Rev. D66, 097502 (2002); B. Bringoltz and M. Teper, Phys. Lett. B645, 383 (2007).

[20] D. Karabali and V.P. Nair, Phys. Rev. D98, 105009 (2018).

[21] M.N. Chernodub, V.A. Goy, A.V. Molochkov and H.H. Nguyen, Phys. Rev. Lett. 121, 191601 (2018).

[22] See, for example, D. Gross, R. Pisarski and L. Yaffe, Rev. Mod. Phys. 53, 43 (1981) and references therein. 